\title{
Numerical Simulation of Incompressible Turbulent Flow using Linear Eddy Viscosity-based Turbulence Models
}

\author{
Sekhar Majumdar", B.N. Rajani**, D.S. Kulkarni* ${ }^{* *}$, and M.B. Subrahmanya** \\ "Nitte Meenakshi Institute of Technology, Bengaluru-560 064 \\ ${ }^{* * N}$ National Aerospace Laboratories, Bengaluru-560 017 \\ Email:essem17@gmail.com
}

\begin{abstract}
The present study focuses on the recent development of an implicit pressure-based finite volume algorithm for numerical solution of Reynolds averaged Navier-Stokes equations (RANS) in an inertial frame of reference for the prediction of unsteady incompressible flow problems. The algorithm uses boundary-conforming, multiblock structured grid with moving boundaries, collocated variable arrangement with momentum equations resolved along cartesian directions, second-order accurate spatial and temporal discretisation schemes for the convective fluxes and a pressure-velocity solution strategy. Effect of turbulence was simulated using appropriate linear eddy viscosity-based turbulence models. The capabilities and limitations of the cost-effective unsteady Reynolds averaged Navier-Stokes (URANS) approach has been demonstrated for few application problems of engineering interest.
\end{abstract}

Keywords: Multi-block boundary-conforming grid, moving boundary, pressure-velocity solution strategy, eddy viscosity-based turbulence models, unsteady flow

\section{INTRODUCTION}

The recent advances of computational fluid dynamics(CFD) in the area of incompressible flows are gradually proving to be invaluable assets for design and analysis of complex problems in the areas of hydrodynamics as well as low-speed aerodynamics. Some important examples include, design of ships, submarines, underwater missiles, off-shore structures, commercial transport aircraft, automobiles, etc. Accurate prediction of turbulent flow around complex structures is of practical interest in the calculation of the drag resistance of the body, in the design of propellers and other appendages, in the analysis of flow-induced noise, and finally, in the determination of the ensuing wake behind the structure.

Specially numerical simulation of unsteady flows with one or more moving boundaries is of great interest for the designers to understand the dynamics of various complex flow situations like the behaviour of an aircraft or a naval vessel during manoeuvers. In these applications, flows are highly nonlinear due to unsteadiness, flow separation, viscous/inviscid, vortex/body or vortex/vortex kind of interactions, transition-to-turbulence or relaminarisation.

Extensive research therefore continues along various fronts to fully integrate CFD capabilities into the design process of ship, automobiles or low-speed transport aircraft. However, considering the constraint of computing resources, Unsteady Reynolds Averaged Navier-Stokes(URANS) methodology coupled with appropriate turbulence models, is often used in practice as the most cost-effective approach to predict the mean flow characteristics of the complex turbulent flow systems. Two major arguments against the use of URANS procedure are the loss of many important details of turbulence interaction due to Reynolds averaging and also that almost all the eddy viscosity-based turbulence models have been designed and calibrated on the basis of mean flow parameters of turbulent shear flows only. On the other hand, in the moderately expensive large eddy simulation (LES) approach, the complex physics of turbulent flow is resolved accurately since the motion of the large scale flow structures involved in momentum and energy transfer processes are resolved numerically from the 3-D unsteady NS equations and the effect of the smallest fine scales of turbulence only are modelled. The present study provides an overview of the work carried out at the CTFD Division, NAL, Bengaluru, during the last twenty years on the development of a robust and accurate generalgeometry finite volume algorithm for CFD analyses of unsteady incompressible flows using URANS approach for turbulence simulation. The capabilities and limitations of the methodology are demonstrated for a few interesting validation test cases.

\section{NUMERICAL METHOD AND TURBULENCE SIMULATION}

\subsection{Governing Equations of the URANS Approach}

In an inertial frame of reference, the Reynolds Averaged Navier-Stokes (RANS) equations and the continuity equation for unsteady incompressible flow with moving boundaries 
may be written in tensor form as following using general non-orthogonal curvilinear coordinates where $j, k$ and $m$ are the summing indices; $\mu$ and $\rho$ are the fluid viscosity and density, respectively; $p$ and $\bar{U}_{i}$ are the time-averaged pressure and cartesian velocity component, respectively; $u_{i}$ is the corresponding fluctuating velocity component due to turbulence and $\dot{x}_{k}$ is the grid velocity component indicating the motion of the body around which the flow is analysed. $J$ is the transformation Jacobian between the cartesian and the curvilinear coordinates and $\beta_{j}^{i}$ and $B_{j}^{i}$ are the relevant geometric coefficients related to the transformation.

Momentum conservation :

$$
\begin{aligned}
& \frac{\partial \bar{U}_{i}}{\partial t}+\frac{1}{J} \frac{\partial}{\partial x_{j}}\left[\begin{array}{c}
\left(\bar{U}_{i}\left(\bar{U}_{k}-\dot{x}_{k}\right) \beta_{k}^{j}\right)-\frac{v}{J}\left(\frac{\partial \bar{U}_{i}}{\partial x_{m}} B_{m}^{j}+\frac{\partial \bar{U}_{k}}{\partial x_{m}} \beta_{i}^{m} \beta_{j}^{k}\right) \\
+\frac{p}{\rho} \beta_{i}^{j}+\overline{u_{i} u_{k}} \beta_{k}^{j}
\end{array}\right]=0 \\
& \text { Mass conservation: } \frac{\partial}{\partial x_{j}}\left(\left(\bar{U}_{k}-\dot{x}_{k}\right) \beta_{k}^{j}\right)=0
\end{aligned}
$$

The Reynolds stress tensor $-\overline{u_{i} u_{k}}$ is evaluated through appropriate turbulence models. The linear Eddy viscosity (LEV)-based models, most widely used in URANS computation of complex flows, assume the Reynolds stress tensor components to be directly proportional to the mean strain rates as follows:

$$
-\overline{u_{i} u_{k}}=\frac{v_{t}}{J}\left(\frac{\partial \bar{U}_{i}}{\partial x_{n}} \beta_{k}^{n}+\frac{\partial \bar{U}_{k}}{\partial x_{m}} \beta_{i}^{m}\right)-\frac{1}{3} \delta_{i k} \overline{u_{m} u_{m}}
$$

where, $\delta_{i j}$ is the Kronecker Delta and the subscript $m$ and $n$ are summing indices. The eddy viscosity $v_{t}$ is evaluated from the relationship with the local turbulence scalars as following :

$$
v_{t}=C_{\mu} E_{s} T_{s}
$$

where $E_{s}$ and $T_{s}$ are appropriate energy scale and time scale, respectively defining the local turbulence level, and $C_{\mu}$ is a model constant. Five different eddy viscositybased turbulence models have been incorporated in the present URANS algorithm. The modelled transport equations for the relevant turbulence scalars, the closure coefficients, the special damping functions, and additional terms for simulation of the near-wall effects in $k$ - $\varepsilon$ model are described.

\subsection{Turbulence Models}

\subsubsection{High Reynolds Number Version of $k$ - $\varepsilon$ Model}

In this model, proposed originally by Launder and

Spalding ${ }^{1}$, the eddy viscosity, $v_{t}$ in Eqn. (3) for momentum transport is computed from a turbulence velocity scale $\left(k^{1 / 2}\right)$ and a turbulence length scale $\left(k^{3 / 2} / \varepsilon\right)$ which are predicted at each point in the flow via solution of the following transport equations for turbulent kinetic energy and its dissipation rate.

$$
D_{t} k=P_{k}-\varepsilon+\nabla \cdot\left(\left(v+\frac{v_{t}}{\sigma_{k}}\right) \nabla k\right)+D
$$

$$
D_{t} \varepsilon=\frac{f_{1} C_{\varepsilon 1} P_{k}-f_{2} C_{\varepsilon 2} \varepsilon}{T}+\nabla \cdot\left(\left(v+\frac{v_{t}}{\sigma_{\varepsilon}}\right) \nabla \varepsilon\right)+E
$$

where the eddy viscosity $v_{\mathrm{t}}$, the turbulent time scale $T$ and the production term $P_{k}$ are expressed as following in which the $S_{i j}$ denotes the mean strain rate. $D_{t}$ represents the total derivative of the relevant flow variable wrt time representing the convective terms

$$
\begin{aligned}
& v_{t}=f_{\mu} C_{\mu} k T ; T=\frac{k}{\varepsilon} ; P_{k}=2 v_{t}\left|S_{i j} S_{i j}\right| ; \\
& S_{i j}=\frac{1}{2}\left(\frac{\partial \bar{U}_{i}}{\partial x_{j}}+\frac{\partial \bar{U}_{j}}{\partial x_{i}}\right)
\end{aligned}
$$

The model constants are $C_{\varepsilon 1}=1.44, C_{\varepsilon 2}=1.92$, $C \mu=0.09$, and the exchange coefficients for the turbulence scalars are $\sigma_{\varepsilon}=1.3$ and $\sigma_{k}=1.0$. In this version, the first near-wall point is assumed to be in the logarithmic layer where the logarithmic law of wall is used for computation of mean velocity and turbulence scalars. Accordingly the damping functions $f_{\mu}, f_{1}$ and $f_{2}$ are set to 1.0 and the special additional terms $D$ and $E$ are set to zero.

\subsubsection{Low Reynolds Number Version of $k$ - $\varepsilon$ Model}

In the low Reynolds number version of the $k-\varepsilon$ model, the near-wall damping functions $f_{1}, f_{2}$ and $f_{\mu}$ are assumed to be exponential functions of the near-wall distance, similar to the Van Driest function proposed for near-wall variation of turbulent length scale in the mixing length model of turbulence. The function $f_{\mu}$ is introduced in the definition of the eddy viscosity $v_{t}$ to mimic the direct effect of the molecular viscosity on the shear stress. These special terms, functions, and the closure coefficients, discussed in detail by Chien ${ }^{2}$ are given below. The values of $k$ and $\varepsilon$ at the wall are set to zero.

$$
\begin{aligned}
& C_{\mu}=0.09, C_{\varepsilon 1}=1.44, C_{\varepsilon 2}=1.92, \sigma_{k}=1.0, \\
& \sigma_{\varepsilon}=1.3, f_{\mu}=1-\exp \left(-0.0115 y^{+}\right), \quad f_{1}=1.0 \\
& f_{2}=1-0.22 \exp \left[-\left(\frac{R_{t}}{6}\right)^{2}\right], y^{+}=\rho \frac{U_{\tau} y}{\mu}, \\
& R_{t}=\rho \frac{k^{2}}{\mu \varepsilon}, \quad D=-2 v \frac{k}{y^{2}}, \quad E=-2 v \frac{\varepsilon}{y^{2}} \exp \left(-0.5 y^{+}\right)
\end{aligned}
$$

\section{$2.2 .3 k$ - $\omega$ Model}

This model uses transport equations of $k$ for the velocity scale and the specific dissipation $\omega(=\varepsilon / k)$ for the length scale. The transport equations ${ }^{3}$ to be solved are given below:

$$
D_{t} k=P_{k}-\beta^{*} k \omega+\nabla \cdot\left(\left(v+\frac{v_{t}}{\sigma_{k}}\right) \nabla k\right)
$$




$$
D_{t} \omega=\alpha P_{k} \frac{\omega}{k}-\beta \omega^{2}+\nabla \cdot\left(\left(v+\frac{v_{t}}{\sigma_{\omega}}\right) \nabla \omega\right)
$$

where the eddy viscosity is given as, $v_{t}=k / \omega$. The model constants are $\alpha=5 / 9, \beta=3 / 40, \beta^{*}=0.09$, and the exchange coefficients are $\sigma_{\omega}=\sigma_{k}=2$. The major advantage of this model is that the equations can be integrated right up to the wall and the finite value of $\omega$ may be derived analytically to be equal to $6 \beta v / y^{2}$ where $y$ is the wall normal distance of the near-wall point and $v$ is the kinematic viscosity of the fluid. However the $k$ - $\omega$ model is found to be extremely sensitive to the turbulence level prescribed at the freestream edge of the boundary layer.

\subsubsection{Shear Stress Transport Model}

The idea behind shear stress transport model proposed by Menter ${ }^{4}$ is to retain the robust and accurate formulation of the Wilcox $k-\omega$ model at the near-wall region, and at the same time, to take advantage of the free stream independence of the $k-\varepsilon$ model in the outer part of the boundary layer. This model is based on the assumption that in adverse pressure gradient flow, the principal turbulent shear stress obeys the same transport equation of turbulent kinetic energy. The transport of $k$ is already represented by Eqn. (5) and the transport equation for $\omega$ is expressed as Eqn. (9) where the last term is a cross derivative term which vanishes for $F_{1}=1$, representing the correct equation for $\omega$ [Eqn. (8)]. When $F_{1}=0$, this cross derivative term does not vanish and using the definition of $\omega$ as $\varepsilon / k$, Eqn. (9) represents the transport equation for $\varepsilon$ in terms of $\omega$ only. $F_{1}$, therefore behaves like a blending function, varying from unity near the wall ( $\omega$ equation) to zero ( $\varepsilon$ equation) as one moves away from the wall. The same blending function is also used to compute the model constants from those of the two different dissipation equations for $\omega$ and $\varepsilon$, respectively.

$$
\begin{aligned}
D_{t} \omega & =\alpha P_{k} \frac{\omega}{k}-\beta \omega^{2}+\nabla \cdot\left(\left(v+\frac{v_{t}}{\sigma_{\omega}}\right) \nabla \omega\right) \\
& +2\left(1-F_{1}\right) \sigma_{\omega 2} \frac{1}{\omega} \frac{\partial k}{\partial x_{j}} \frac{\partial \omega}{\partial x_{j}}
\end{aligned}
$$

The eddy viscosity is computed using the following formula and using a limiter based on the proportionality of shear stress to the turbulence energy in case of adverse pressure gradient flow:

$$
v_{t}=\min \left(\frac{k}{\omega}, \frac{a_{1} k}{\Omega F_{2}}\right)
$$

The blending functions $F_{1}$ and $F_{2}$ are expressed as hyperbolic functions, varying from unity at wall to zero near the free-stream edge; $\Omega$ is the magnitude of the mean vorticity and $C D_{k-\omega}$ represents the cross derivative term appearing at the end of the rhs of Eqn. (9).

$$
F_{1}=\tanh \left(\arg _{1}^{4}\right)
$$

where

$$
\arg _{1}=\min \left[\max \left(\frac{\sqrt{k}}{0.09 \omega y} ; \frac{500 v}{y^{2} \omega}\right) ; \frac{4 \rho \sigma_{\omega 2} k}{C D_{k-\omega} y^{2}}\right]
$$

and

$$
F_{2}=\tanh \left(\arg _{2}^{2}\right)
$$

where

$$
\arg _{2}=\max \left(2 \frac{\sqrt{k}}{0.09 \omega y} ; \frac{500 v}{y^{2} \omega}\right)
$$

Any model constant $\phi$ is also interpolated with the blending function $F_{1}$ between the corresponding values $\phi_{1}$ for the $k-\omega$ and $\phi_{2}$ for the $k-\varepsilon$ equation as:

$\phi=F_{1} \phi_{1}+\left(1-F_{1}\right) \phi_{2}$

The model constants of the $k-\omega$ equation are:

$$
\begin{aligned}
& \sigma_{k 1}=1.176, \sigma_{\omega 1}=2.0, \beta_{1}=0.075, \beta^{*}=0.09, \\
& \kappa=0.41 \text { and } \alpha_{1}=\beta_{1} / \beta^{*}-\kappa^{2} /\left(\sigma_{\omega 1} \sqrt{ } \beta^{*}\right)
\end{aligned}
$$

and the model constants of the $k-\varepsilon$ equation are :

$$
\begin{aligned}
& \sigma_{k 2}=1.0, \sigma_{\omega 2}=1.168, \beta_{2}=0.0828, \beta^{*}=0.09, \\
& \kappa=0.41 \text { and } \alpha_{2}=\beta_{2} / \beta^{*}-\kappa^{2} /\left(\sigma_{\omega 2} \sqrt{ } \beta^{*}\right)
\end{aligned}
$$

\subsubsection{Spalart-Allmaras Model}

This model ${ }^{5}$ solves a single transport equation [Eqn. (10)] for a high Reynolds number eddy viscosity variable $\widetilde{v}_{t}$ which when multiplied by another local function $f_{v l}$ gives the eddy viscosity $v_{t}$.

$$
\begin{aligned}
D_{t} \tilde{v}_{t}= & c_{b 1} \tilde{v}_{t} \tilde{S}-c_{w 1} f_{w}\left(\frac{\tilde{v}_{t}}{d}\right)^{2} \\
& +\frac{1}{\sigma}\left(\nabla \cdot\left(\left(v+\tilde{v}_{t}\right) \nabla \tilde{v}_{t}\right)+c_{b 2} \nabla^{2} \tilde{v}_{t}\right)
\end{aligned}
$$

where, $d$ is the distance of a grid node to the nearest surface. The eddy viscosity defined by the auxiliary function is given by

$$
v_{t}=\tilde{v}_{t} f_{v 1} ; \quad f_{v 1}=\frac{\chi^{3}}{\chi^{3}+c_{v 1}^{3}} ; \chi=\frac{\tilde{v}_{t}}{v}
$$

The absolute value of the vorticity $S$ in the production term and the blending function $f_{w}$ in Eqn. (10) are given by the following functions

$$
\begin{aligned}
& \tilde{S}=S+\frac{\tilde{v}_{t}}{\kappa^{2} d^{2}} f_{v 2} ; \quad f_{v 2}=1-\frac{\chi}{1+\chi f_{v 1}} ; \\
& S=\sqrt{2 \Omega_{i j} \Omega_{i j}} ; \Omega_{i j}=\frac{1}{2}\left(\frac{\partial U_{i}}{\partial x_{j}}-\frac{\partial U_{j}}{\partial x_{i}}\right)
\end{aligned}
$$




$$
f_{w}=g\left[\frac{1+c_{w 3}^{6}}{g^{6}+c_{w 3}^{6}}\right]^{1 / 6} ; g=r+c_{w 2}\left(r^{6}-r\right) ; \quad r=\frac{\tilde{v}_{t}}{\tilde{S} \kappa^{2} d^{2}}
$$

The closure coefficients are; $c_{b 1}=0.1355, c_{b 2}=0.622$, $c_{v 1}=7.1, \sigma=2 / 3, c_{w 1}=\frac{c_{b 1}}{\kappa^{2}}+\frac{\left(1+c_{b 2}\right)}{\sigma}, c_{w 2}=0.3, c_{w 3}=2.0$ and $\kappa=0.41$. The value of $\widetilde{v}_{t}$ at the wall is set to zero and in the free stream, usually very small values are specified for $\widetilde{v}_{t}$.

\subsection{6 $k-\varepsilon-\overline{v^{2}}-f(V 2 F)$ Model}

The V2F model proposed by Durbin ${ }^{6}$ is an approximate version of the Reynolds stress transport model which takes care of the near-wall anisotropy of the normal stresses. In this model, unlike the conventional two equation models, the wall-normal component $\left(\bar{v}^{2}\right)$ of the turbulence energy is assumed to be a more appropriate velocity scale than the turbulence energy $(k)$ itself in the definition of eddy viscosity. Even for the turbulence time scale also, the model uses a limiter which computes the time-scale as $k / \varepsilon$ in the zone far from the wall as in the conventional two-equation models whereas near the wall, the Kolmogorov scale depending on the molecular viscosity is used as a more appropriate turbulent length scale. The transport equation for $\bar{v}^{2}$ [Eqn. (11)] takes care of the near-wall anisotropy of the Reynolds stresses where the production term $k f$ accounts for the redistribution of turbulence intensity from the stream-wise to the stream-normal component. The non-local character of the modelled redistribution term $k f$ is considered by solving an elliptic (Helmholtz type) relaxation equation for the variable $f$ derived from the second moment closure equation for the Reynolds stress. The transport equation for $\bar{v}^{2}$ and $f$ are as follows:

$$
\begin{aligned}
& D_{t} \overline{v^{2}}=k f-6 \frac{\overline{v^{2}}}{k} \varepsilon+\nabla \cdot\left(\left(v+v_{t}\right) \nabla \overline{v^{2}}\right) \\
& L^{2} \nabla^{2} f-f=\frac{1}{T}\left[\left(C_{1}-6\right) \frac{\overline{v^{2}}}{k}-\left(C_{1}-1\right) \frac{2}{3}\right]-C_{2} \frac{P_{k}}{k}
\end{aligned}
$$

where the eddy viscosity $v_{t}$, the turbulent length scale $L$ and time scale $T$ are expressed as

$$
\begin{aligned}
& v_{t}=C_{\mu} \overline{v^{2}} T ; L=C_{L} \max \left(\frac{k^{3 / 2}}{\varepsilon} ; C_{\eta}\left(\frac{v^{3}}{\varepsilon}\right)^{1 / 4}\right) ; \\
& T=\max \left(\frac{k}{\varepsilon} ; 6\left(\frac{v}{\varepsilon}\right)^{1 / 2}\right)
\end{aligned}
$$

The closure coefficients are; $C_{\varepsilon 1}=1.4\left(1+0.045 \sqrt{k / \sqrt{v^{2}}}\right)$, $C_{\varepsilon 2}=1.9, C_{\mu}=0.22, C_{1}=1.4, C_{2}=0.3, \sigma_{k}=1, \sigma_{\varepsilon}=1.3$, $C_{L}=0.25, C_{\eta}=85$.

In this model, the transport equations for $k$ and $\varepsilon$ [Eqns (5) \& (6)] are also to be solved along with Eqns (11) \& (12) for $\bar{v}^{2}$ and $f$. Since no adhoc damping function is required for eddy viscosity or turbulence dissipation, the damping functions, $f_{1}$ and $f_{2}$ are set to 1.0 and the special additional terms $D$ and $E$ in Eqns (3) \& (4) are set to zero. The value of all the turbulence scalars except $\varepsilon$ is set to zero on the wall and the value of $\varepsilon$ at the wall is assumed to be $2 k v / y^{2}$, as derived from the balance of the diffusion and dissipation term of the $k$-equation at the near-wall region.

\subsection{Numerical Solution of the Finite Volume Equation}

The relevant Navier-Stokes equation [Eqn. (1)] is first transformed to corresponding finite volume equation in terms of surface flux balance for each control volume using the Gauss divergence theorem. An implicit predictorcorrector method based on a pressure-velocity solution strategy is used for numerical solution of the finite volume equation system. Second-order accurate central difference or higher-order, low-diffusion upwind schemes are used for spatial discretisation of the convective fluxes, whereas the temporal derivatives are discretised using the secondorder accurate three-level fully implicit scheme.

Using the relevant geometric factors, appropriate discretisation schemes, and linearisation of the source terms, the flux balance equations to be solved in the predictor step, for momentum and turbulence scalars are expressed in a generalised implicit manner as follows:

$$
\begin{aligned}
& \left(1.5 \varphi_{P}^{n+1}+0.5 \varphi_{P}^{n-1}-2 \varphi_{P}^{n}\right) \Delta V / \Delta t \\
& =\sum A_{n b} \varphi_{n b}^{n+1}+S U-A_{P} \varphi_{P}^{n+1}
\end{aligned}
$$

where $A_{P}=\sum A_{n b}-S P$; the coefficient $A_{n b}$ represents the combined effect of convection and diffusion at the six faces of a hexahedral computational cell ; $S U$ and $S P$ are the components of the linearised source term, $\Delta V$ is the cell volume and $\Delta t$ is the time step size. In the corrector step, the continuity equation is also transformed to a similar linearised equation for pressure correction in the form of Eqn. (13). The corrections for pressure and velocity field obtained are added to the pressure and the momentumsatisfying velocities at the cell centres and the cell faces, obtained at the predictor step. The derivation of Eqn. (13) and the decoupled iterative procedure to handle the pressurevelocity link are given in other research reports ${ }^{7-9}$. The system of linearised Eqn. (13) is solved at each outer iteration level using the strongly implicit procedure of Stone ${ }^{10}$. 


\section{RESULTS AND DISCUSSION}

\subsection{Laminar Flow Past a Bluff Body Mounted on the}

\section{Lower Wall of a Plane Channel}

This test case is chosen to demonstrate the predictive capability of the present URANS algorithm for non-periodic, time-dependent laminar flows. To study unsteady flow separation on a bluff body mounted on the lower wall of a plane channel, flow visualisation experiments were conducted ${ }^{11}$ using laser-induced fluoroscence (LIF) technique in a special water tunnel consisting of a two-component glass channel where the required flow velocity-time variation [Fig. 1(a)] at the tunnel inlet is maintained by a servomotor system. The test plane is illuminated by a 2-D laser sheet and the image of the Fluorescent Sodium dye introduced on the body surface, is captured by a CCD camera at different instants of time during the piston motion. The URANS computation domain is bounded by the horizontal channel top wall, the test body geometry for the channel lower wall, an inflow and outflow plane at a distance of $8 C$ on either side, where $C$ is the channel height. Close view of the H-Grid $(191 \times 81)$ near the bluff body, used for computation is shown in Fig. 1(b). The algorithm uses central difference scheme for convective fluxes and secondorder time discretisation with time step size $(\Delta t)$ of 0.002 units. Figure 1(c) compares the computed instantaneous streamlines to the flow visualisation pictures at three different time instants starting from rest. Reasonably good agreement is observed between the computation and measurement for the instant and the location of the inception of unsteady flow separation and also for the size of the separation bubble growing with time.

\subsection{Turbulent Flow Past a Symmetric Aerofoil at High Angles of Attack}

At angles of attack near or beyond the stall angle of an aerofoil, the flow invariably separates from the suction surface, leading to periodic vortex shedding, and eventually, generates a wake consisting of multiple vortical flow structures which can be captured through solution of unsteady flow equations only. The present computation ${ }^{12}$ uses a twoblock O-grid [Fig. 2(a)], consisting of $320 \times 100$ control volumes, with the far-field placed at a radius of $15 \mathrm{C}$ and the minimum wall normal distance is maintained at around $8 \times 10^{-6} C$, where $C$ is the aerofoil chord length. The third order accurate QUICK ${ }^{13}$ scheme and second-order accurate three-level implicit scheme have been used for the spatial and temporal discretisation of convective fluxes. Figure 2(b) shows the typical flow boundary conditions, where the far-field is treated either as an inflow or an outflow depending on the sign of the convective flux on the relevant face. Convective boundary condition is used for outflow boundaries. In the multi-block computation environment, every block-interface (cut) boundary is provided with one overlapping control volume on either side of the cut for appropriate transfer of the solution from the neighbouring block. Figure 3 shows the typical instantaneous particle traces and vorticity contours for three different angles of attacks, computed using the S-A turbulence model ${ }^{5}$. The figures clearly show the distinct difference in the vortical structure of the wake flow as the angle of attack $(\alpha)$ changes from $25^{\circ}$ to $90^{\circ}$. At $\alpha=25^{\circ}$, a small trailing edge vortex is shed from the suction surface of the aerofoil and as $\alpha$ increases to $50^{\circ}$, a large clockwise vortex is formed covering a large part of the suction surface indicating significant enhancement of the lift coefficient. Finally at $\alpha=90^{\circ}$, the aerofoil behaves almost like a plate normal to the flow. A very large anticlockwise vortex, covering almost the full blockage area behind the aerofoil, is shed from the aerofoil surface, eventually forming a typical vortex street further downstream in the wake region.

The instantaneous aerodynamic coefficients are calculated from the integration of the tangential wall shear stresses and the wall-normal pressure forces over the whole aerofoil surface. Table 1 shows the sensitivity of the turbulence model on the computed mean aerodynamic coefficients and the Strouhal number at $\alpha=90^{\circ}$. In spite of good qualitative agreement, significant scatter is observed in both computation results as well as in the measurement data reported by different researchers ${ }^{14,15}$.

\subsection{Turbulent Flow Past a Pitching NACA 0012 Aerofoil}

Flow past a pitching NACA0012 aerofoil is another example of a periodic unsteady flow caused by the sinusoidal motion of the aerofoil surface. This test case ${ }^{16}$ validates the accuracy and adequacy of the present URANS algorithm, specially with moving boundary conditions, against the accurate measurement data ${ }^{17}$ reported in literature. The

Table 1. Mean aerodynamic coefficients and Strouhal number for NACA0012 at $\alpha=90^{\circ}$

\begin{tabular}{|c|c|c|c|c|c|c|c|c|}
\hline & \multicolumn{4}{|c|}{ Present Computation } & \multicolumn{3}{|c|}{$\begin{array}{l}\text { Computation } \\
\text { Shur, et al }\end{array}$} & \multirow{2}{*}{$\begin{array}{c}\text { Measurement } \\
\text { Hörner }\end{array}$} \\
\hline & Low $\operatorname{Re} k-\varepsilon$ & $k-\omega$ & SST & SA & V2F & 2D & 3D & \\
\hline Lift, $C_{l}$ & 0.060 & 0.077 & 0.053 & 0.096 & 0.063 & 0.10 & 0.11 & 0.07 \\
\hline Drag, $C_{d}$ & 2.741 & 2.977 & 3.155 & 2.762 & 2.753 & 2.84 & 2.54 & 2.05 \\
\hline $\begin{array}{l}\text { Strouhal } \\
\text { Number }\end{array}$ & 0.098 & 0.117 & 0.107 & 0.137 & 0.117 & & & \\
\hline
\end{tabular}




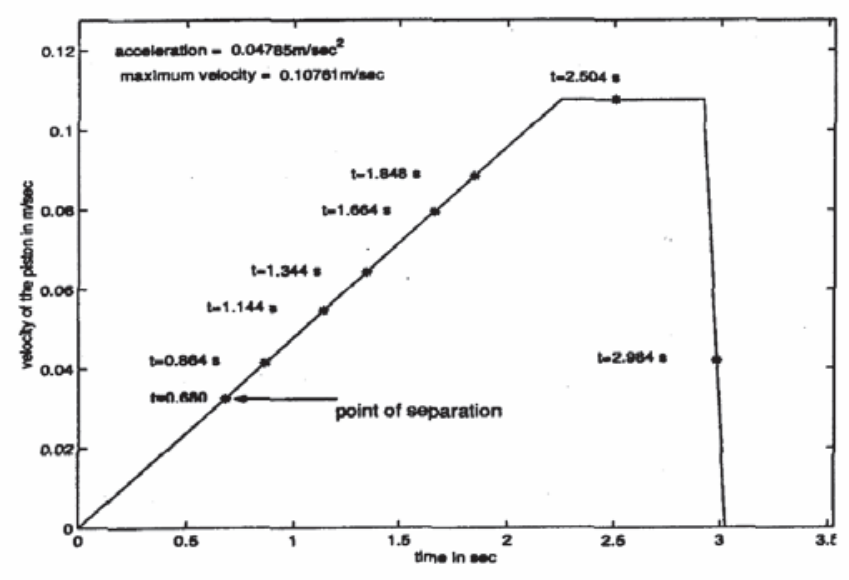

(a)

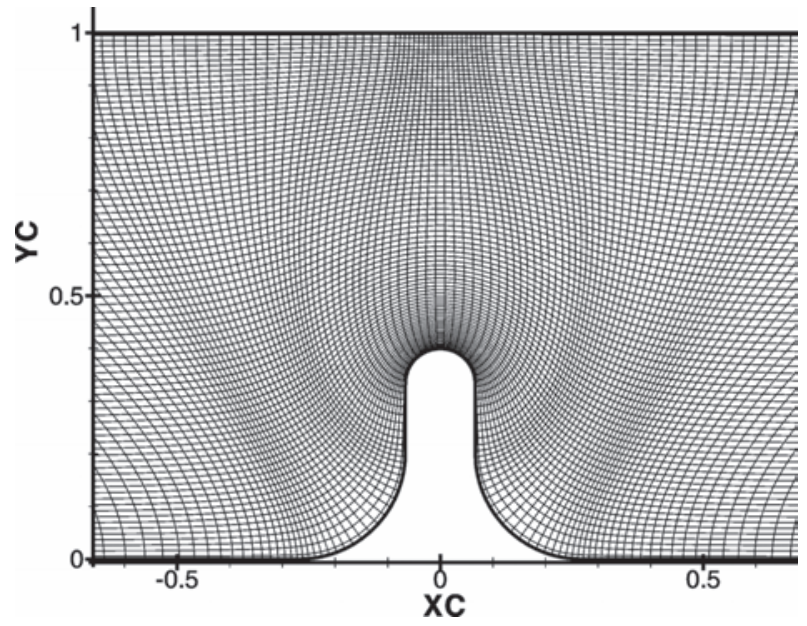

(b)
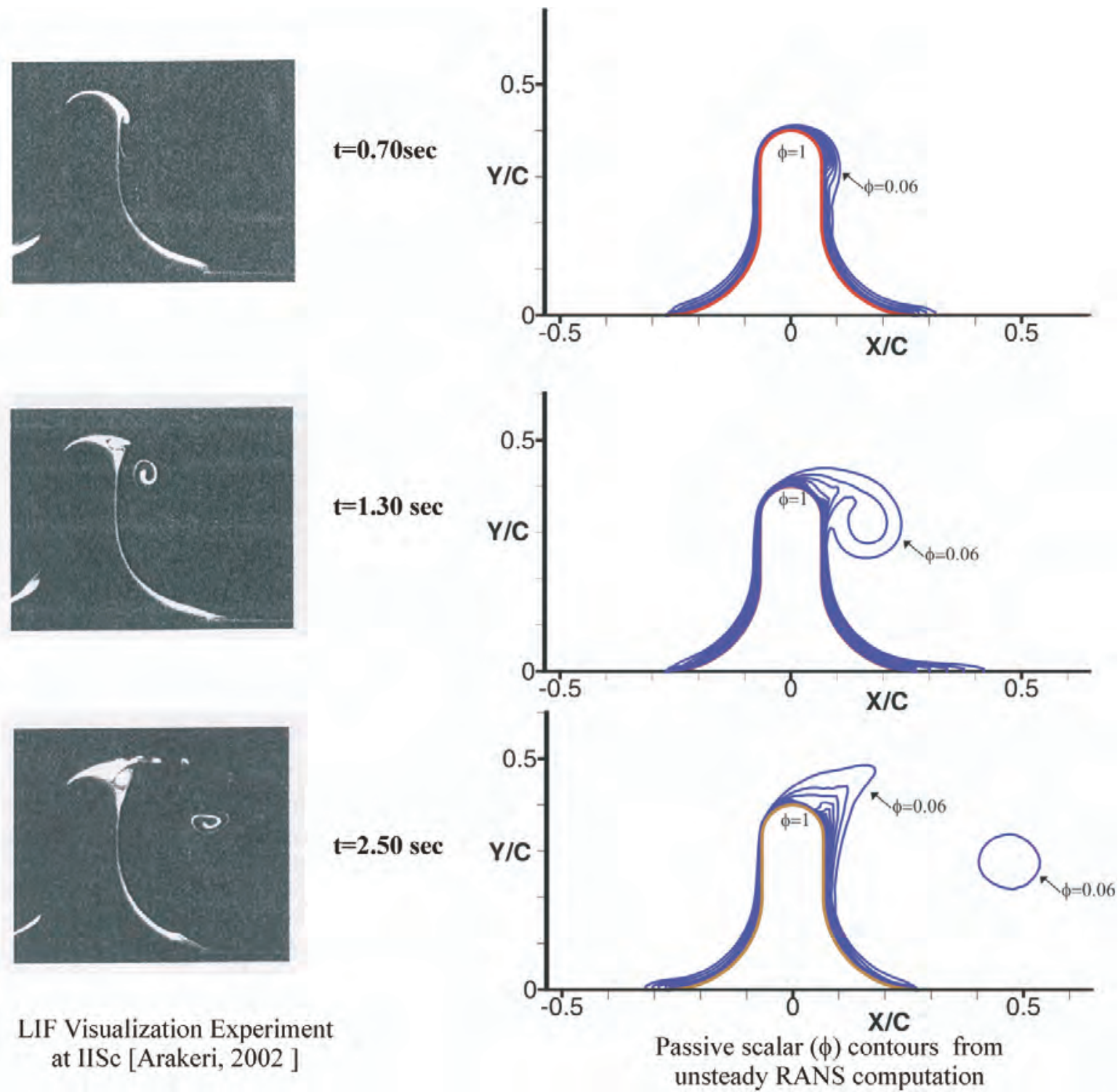

LIF Visualization Experiment at IISc [Arakeri, 2002 ]

(c)

Figure 1. Unsteady laminar flow past a bluff body mounted on the lower wall of a channel;. (a) Time-velocity variation in visualisation study, (b) Computational grid $(191 \times 81)$, and (c) Comparison between present computation and flow visualisation. 

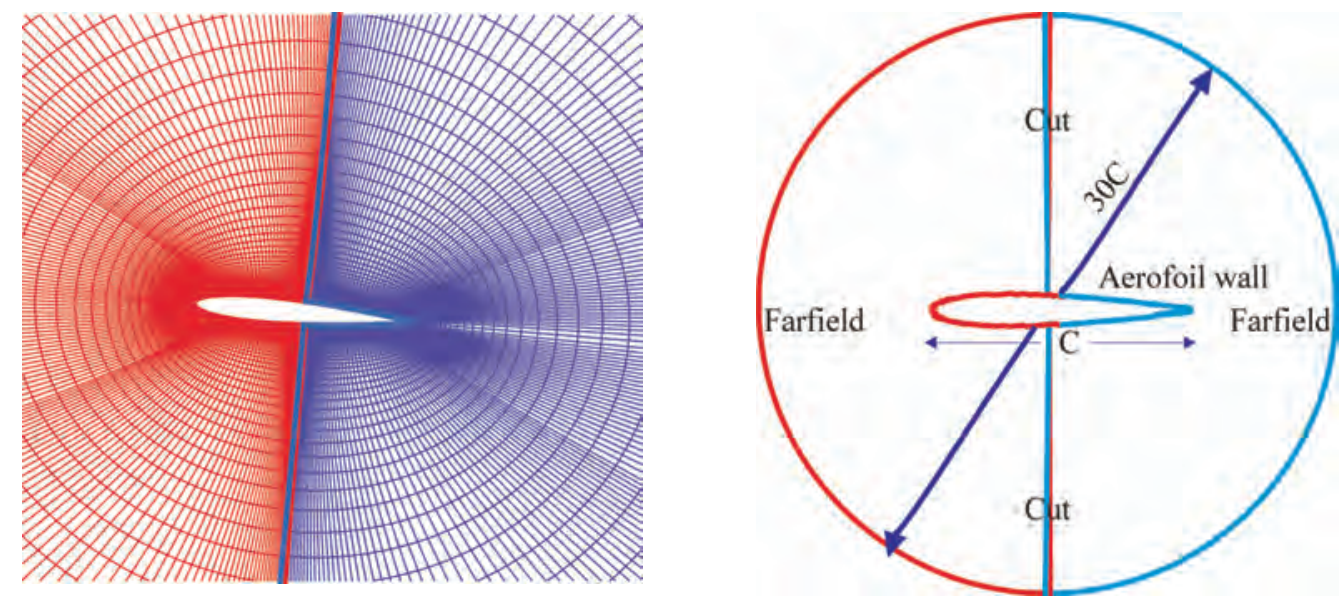

Figure 2. Grid and boundary condition used for aerofoil flow computation; (a) zoomed view of 2 block O-grid and (b) boundary condition for flow computation.
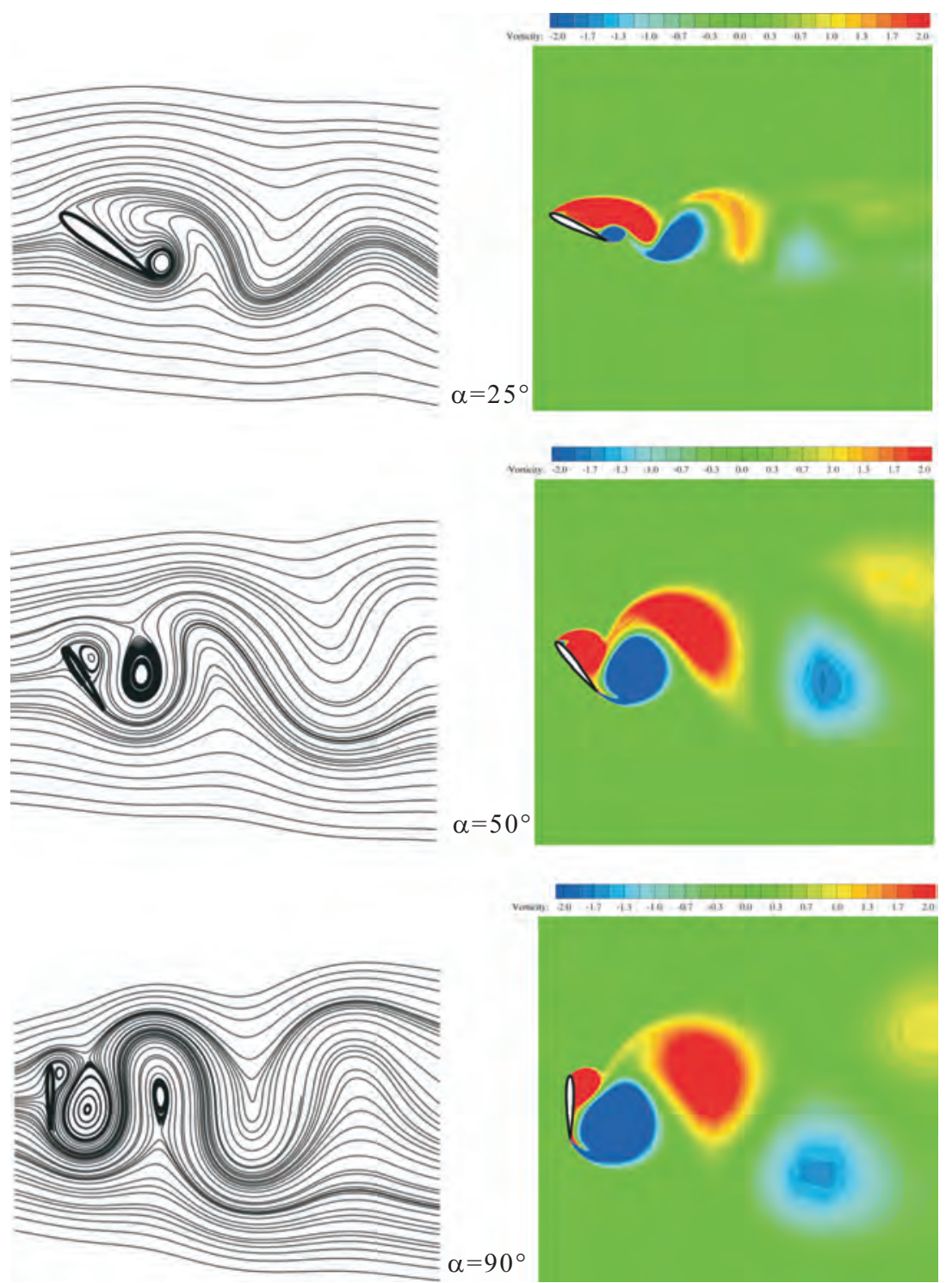

Figure 3. Instantaneous particle traces and vorticity contours for flow part NACA0012 aerofoil at different angles of attack (SA Turbulence model at Reynolds number $=10^{6}$ ). 

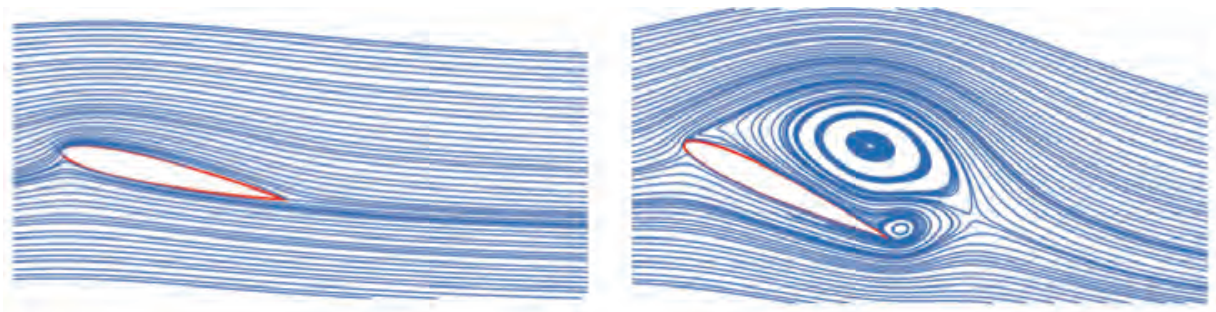

PARTICALE TRACES

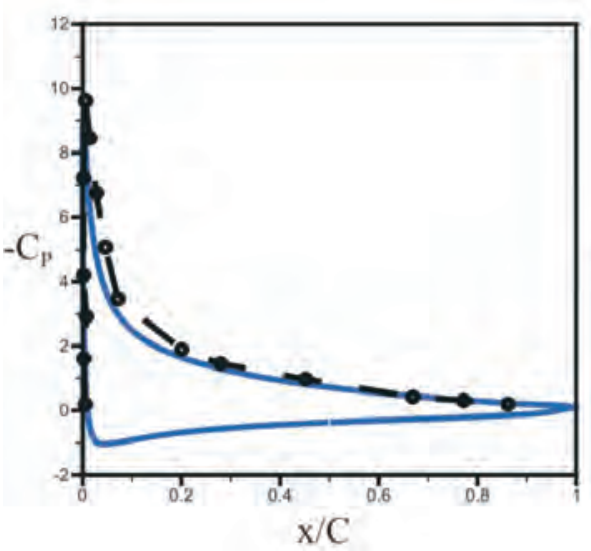

$\alpha=18^{\circ} \uparrow$

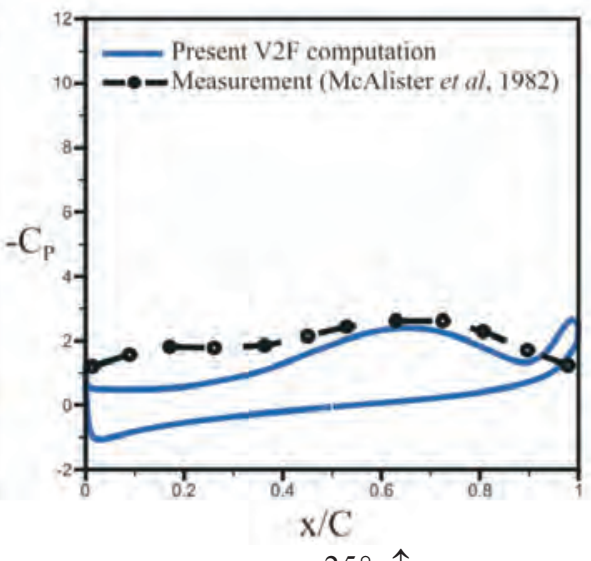

$\alpha=25^{\circ} \uparrow$
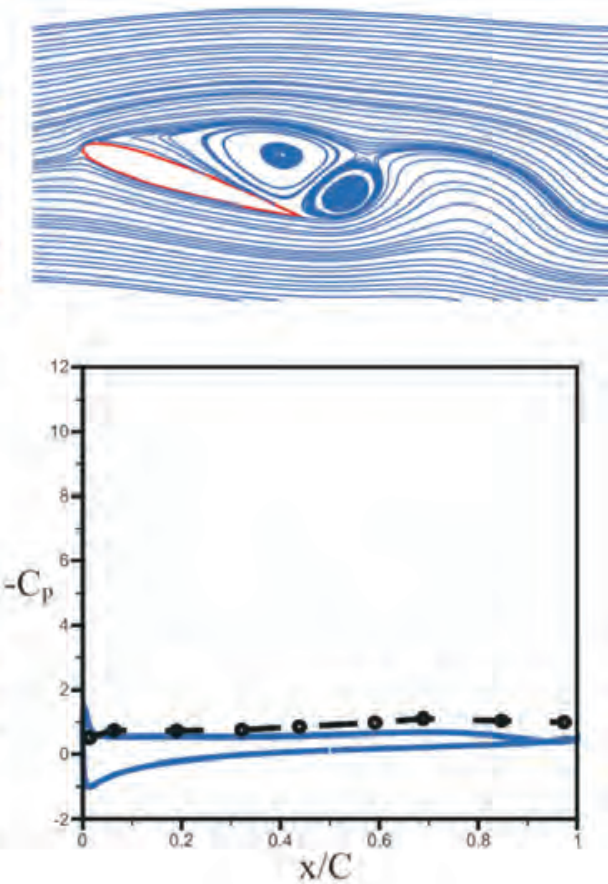

$\alpha=17.5^{\circ} \downarrow$

(a)

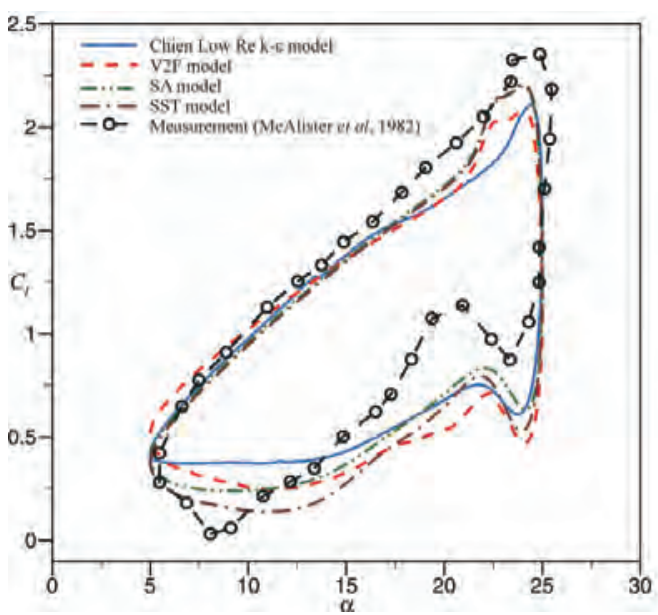

(b)

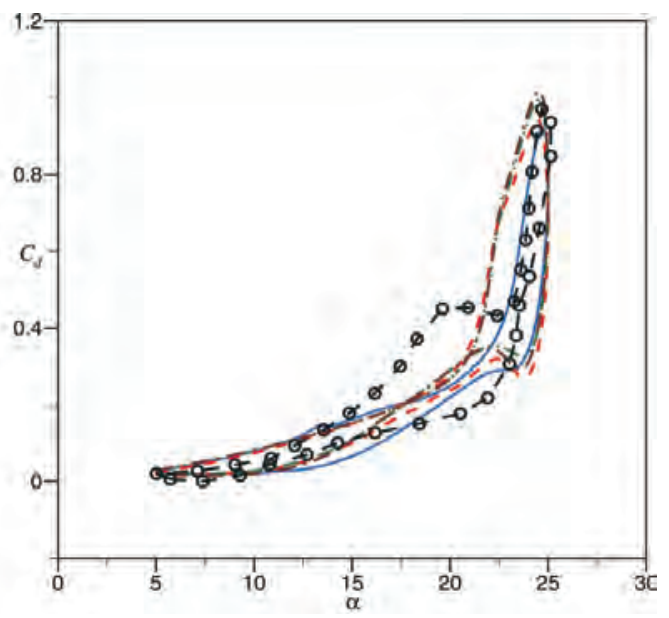

(c)

Figure 4. Turbulent flow past a pitching NACA 0012 aerofoil $\left(\operatorname{Re}=10^{6}, k=0.15\right.$ and $\left.\Delta t=0.05\right)$ : (a) instantaneous particle traces and surface pressure distribution, (b) lift coefficient, and (c) drag coefficient.

aerofoil is subjected to a pitching motion about the quarterchord point, defined and $k\left(=\omega C / U_{\infty}\right)=0.15$ is the reduced frequency of the oscillatory motion where $\omega$ is the physical frequency. The same numerical grid and convective flux discretisation scheme, used for stationary aerofoil test case described in the previous sub-section, are employed in the pitching aerofoil flow situation. Figure 4 (a) shows the instantaneous particle traces and the history of the surface pressure on the aerofoil at three different instants of the pitching cycle. The prediction of the upper surface pressure agrees reasonably well with the measurement data ${ }^{17}$ for different angles of attack encountered during the upstroke and downstroke of the pitching motion of the aerofoil.

At maximum value of $\alpha=25^{\circ}$, whole of the suction surface is observed to be covered by a large clockwise vortex with a small counter-clockwise vortex near the trailing edge which, during the downward motion, eventually pulls the large hysteresis vortex towards the trailing edge and hence reduces the vortex strength on the suction surface. As the value of $\alpha$ decreases during the downward motion, the large single vortex breaks into multiple small vortices, leading to sudden reduction of the suction pressure on the upper surface, and hence, to drastic loss of lift. Figures 4(b) and 4 (c) show the dynamic hysteresis loops for the lift and drag coefficients computed using different turbulence models. Results using the SA and SST turbulence models are observed to be in better agreement with the measurement data. The double peaking of $C_{l}$ during the start of the 

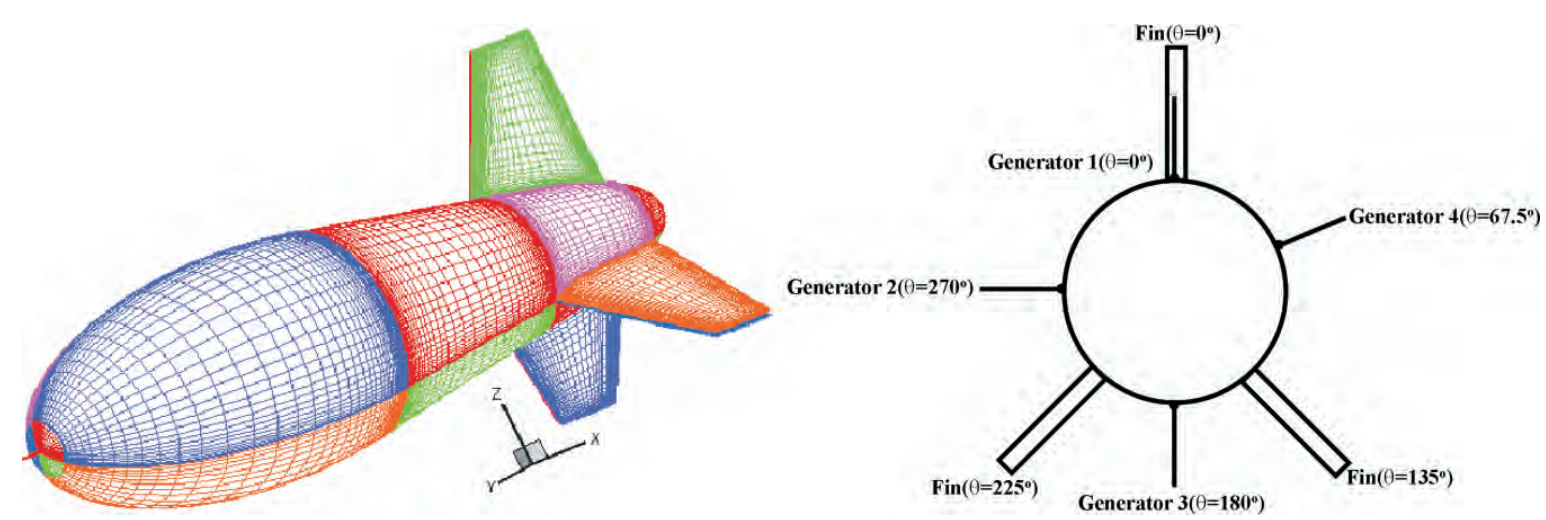

(a)
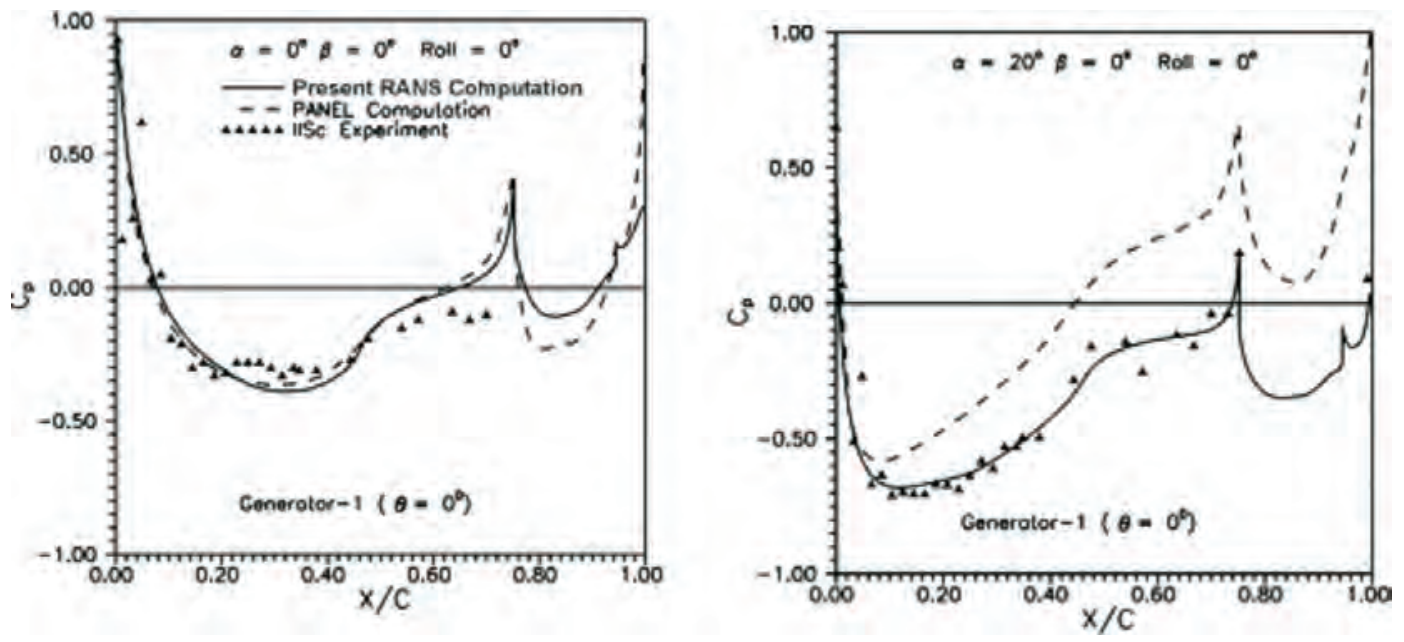

(b)

Figure 5. Turbulent flow around aerostat balloon with fins $\left(\operatorname{Re}=1.5 \times 10^{7}\right)$ : (a) multi-block surface grid for Aerostat flow computation and (b) Surface pressure distribution along the top generator of the hull surface.

return downward stroke of the aerofoil, has been reasonably captured by all the models. However quantitative disagreement observed at some regions, may perhaps be attributed to the uncertainties and inadequacies of the eddy-viscositybased turbulence models used.

\subsection{Turbulent Flow Past an Aerostat Balloon}

The objective of selecting this steady flow problem is to demonstrate the capability of the multi-block parallel version of the present URANS algorithm even for prediction of steady three-dimensional turbulent flow past any complex arbitrary shaped geometry. This problem is about prediction of a high $\operatorname{Re}\left(\operatorname{Re}=1.5 \times 10^{7}\right)$ turbulent flow around an aerostat balloon configuration consisting of an axisymmetric hull generated from a smooth aerodynamic shape along with three fins of aerofoil (NACA0018) cross-section, attached near the tail end of the hull as control surfaces. The computed surface pressure distribution ${ }^{18}$ along the top surface generator line $\left(\theta=0^{\circ}\right.$ in Fig. 5 (a)) of the aerostat, obtained from the present URANS algorithm, is validated against corresponding potential flow computation for the same configuration, using Panel method ${ }^{19}$, reported by Narayan, et al. and also against the measurement data obtained from IISc wind tunnel tests $^{20}$ on a $1 / 7^{\text {th }}$ scaled model.

Circumferentially stacked 2-D grid is used in the domain outside the fin region whereas the grids [Fig. 5(a)] on the fin-surface are laid out separately using a purely algebraic procedure. QUICK scheme ${ }^{13}$ with deferred correction approach $^{21}$ and $k$ - $\varepsilon$ turbulence model with standard wall function ${ }^{1}$ have been used for the flow computations. The computation domain covering $300 \times 82 \times 92$ control volumes has been decomposed into 18 blocks computed in parallel using six Pentium processors of NAL parallel machine Flosolver Mk5, each covering three consecutive blocks along circumferential direction. Figure 5(b) shows the pressure distribution along the top surface generator line of the hull at two different angles of attack. The comparison clearly shows reasonable agreement of the measurement data to the present RANS solution and also the expected large discrepancies between measurement data and the Panel code results at high angle of attack.

\subsection{Turbulent Flow Past an Underwater Body with Stern-end Appendages}

This test case ${ }^{22}$ also deals with prediction of three -dimensional turbulent flow around an axisymmetric hull 

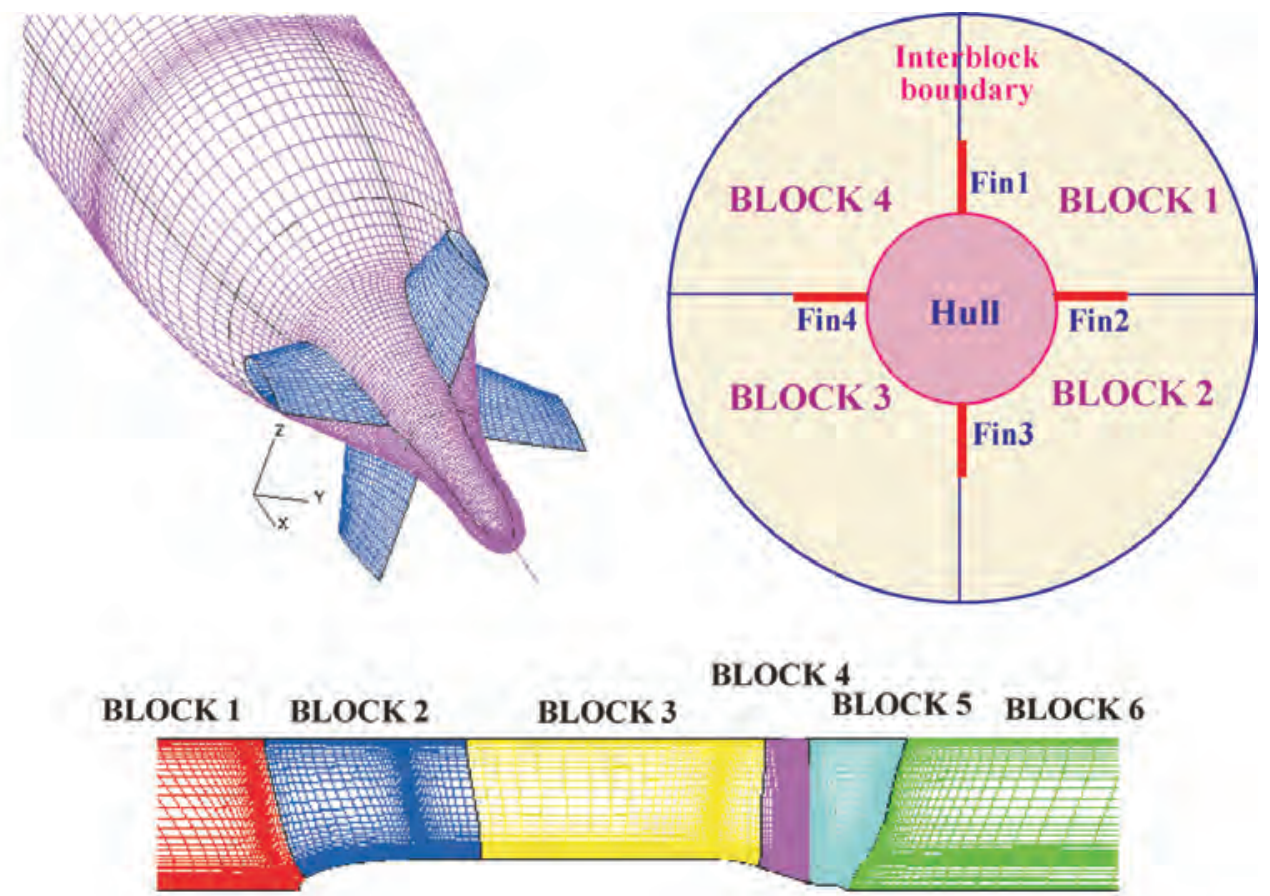

(a)

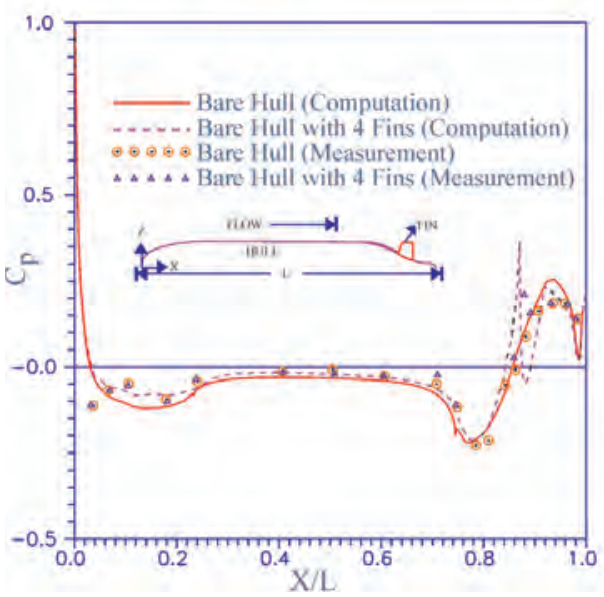

(b)

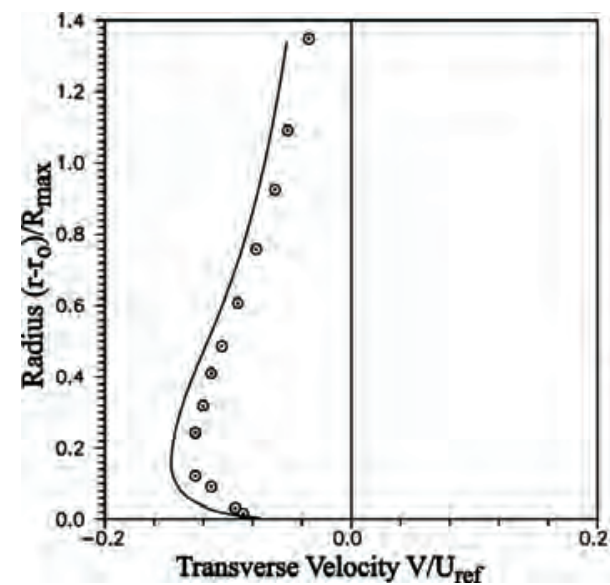

(d)

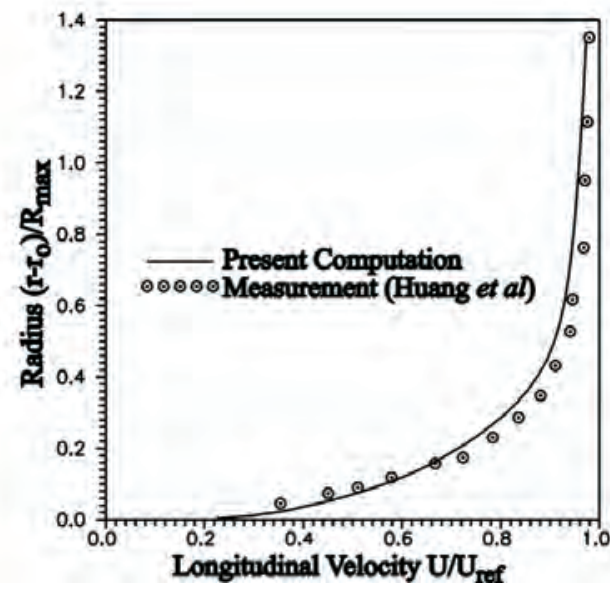

(c)

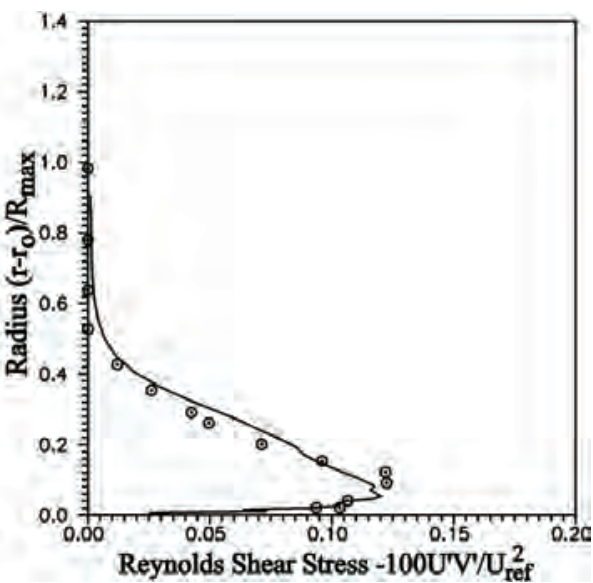

(e)

Figure 6. Turbulent flow around a DARPA Suboff model with stern-end appendages $\left(\operatorname{Re}=1.2 \times 10^{7}\right)$ : (a) Block arrangement and Surface grid for the hull with fins, (b) Surface pressure distribution, (c) U velocity profile (X/L =0.904 ), (d) V-velocity profile $(\mathrm{X} / \mathrm{L}=\mathbf{0 . 9 0 4})$, and (e) Reynolds Stress profile $(\mathrm{X} / \mathrm{L}=\mathbf{0 . 9 0 4})$. 
of the DARPA Sub-off model of submarine consisting of a forebody, a parallel mid-body section, and an afterbody with four radial fins of NACA0020 aerofoil cross- section attached at the stern end of the hull. Detailed hot-film anemometer measurements were reported ${ }^{23}$ for this case from the David Taylor Model Basin Research Group,USA. A differential-algebraic grid generation procedure ${ }^{24}$, developed at the CTFD Division, NAL was employed to generate structured, boundary-fitted grid required for the present problem. The computational domain was divided into 24 blocks, four along the circumferential and six along the longitudinal direction [Fig. 6(a)]. A H-O grid topology is used with total number of 245 control volumes (CV) along longitudinal, $80 \mathrm{CV}$ along radial and $82 \mathrm{CV}$ along tangential directions. A close view of the surface grid near the hullfin intersection is also shown in Fig. 6(a). The present prediction $^{22}$ is compared in Fig. 6(b) with the measurement data for surface pressure along the vertical meridional plane of the hull with fins.

The disagreement between computation and measurement data near the stern end, may be attributed partly to the

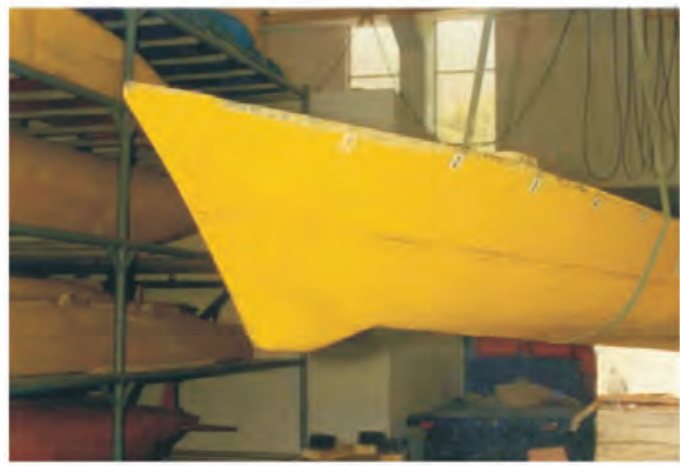

(a)

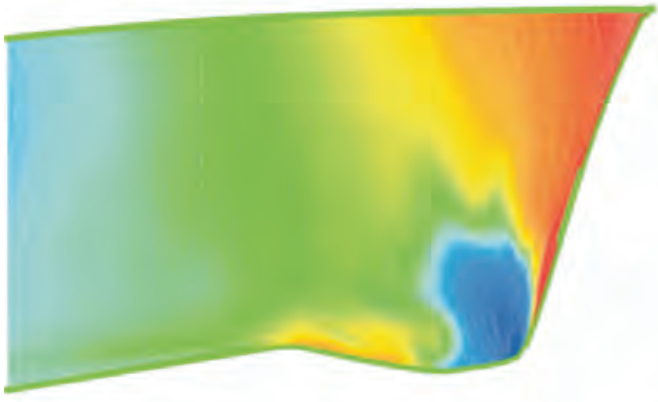

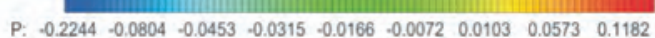

(b) inaccuracy in the geometry-prescription near the strong curvature zone of the stern-end and partly to the well known inadequacy of the $\mathrm{k}-\varepsilon$ models in the adverse pressure gradient region. The present prediction is also compared to the measurement data ${ }^{23}$ in Figs. 6(c) to 6(e) for the transverse profiles of the longitudinal velocity, transverse velocity and also the Reynolds shear stress for the bare hull at a longitudinal station $X / L=0.904$ and the agreement is observed to be reasonably good.

\subsection{Turbulent Flow Past a Ship Hull with Bow- mounted Sonar Dome}

This is an interesting example of ship viscous flow which is assumed to be steady and also symmetric around the vertical mid-plane of the configuration. This turbulent flow problem for complex geometry is predicted using the present URANS algorithm and validated against corresponding measurement data on the drag forces on the dome surface only, obtained from a towing tank test on a scaled down model of the configuration. The geometry of the hull with bow-mounted sonar dome is specified by NSTL,

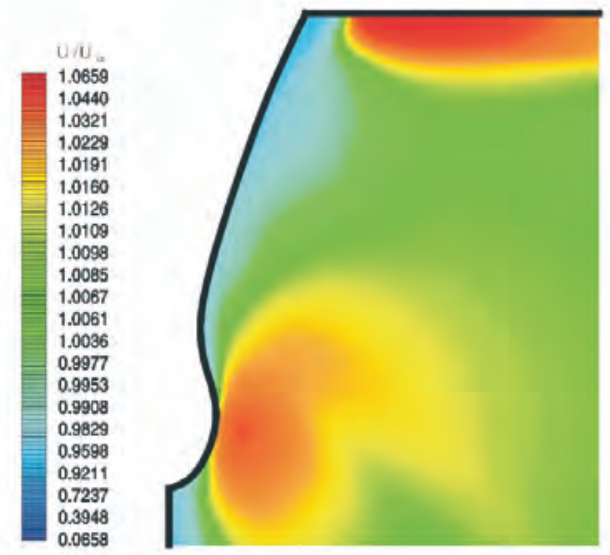

(c)

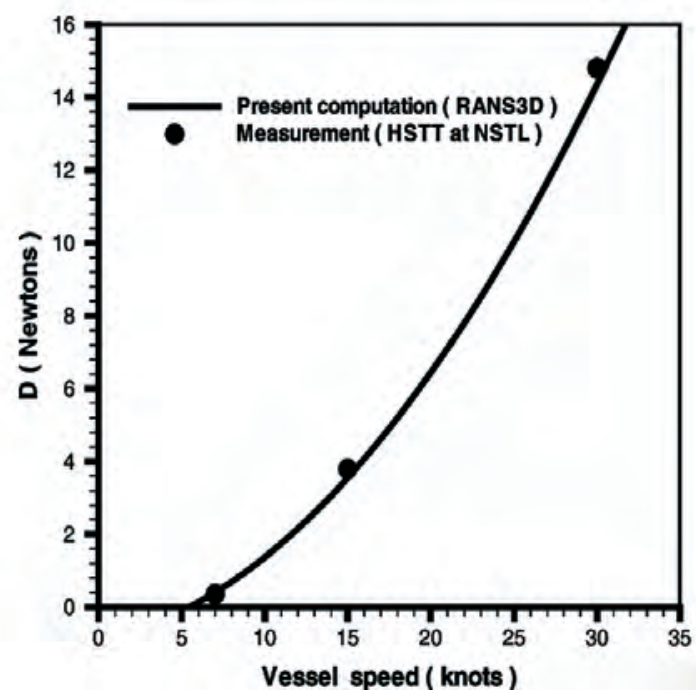

(d)

Figure 7. Turbulent flow around a ship hull with bow-mounted sonar dome: (a) hull model for resistance test at HSTT, NSTL, (b) pressure contours on hull surface, (c) longitudinal velocity contours at $\mathrm{X} / \mathrm{L}=\mathbf{0 . 0 7 9 4}$, and (d) drag force on the sonar dome. 
Visakhapatnam, and a differential-algebraic hybrid grid generation methodology was used to generate a stacked quasi-3-D grid consisting of vertical parallel planes in the form of a H-O grid topology $(109 \times 109 \times 65)$. Flow computations have been carried out for three different hull speeds of 7 knots, 15 knots, and 30 knots and detailed results are documented ${ }^{25}$. The stretching factor and number of grids along the transverse direction are so chosen that the near-wall distance in wall coordinate $\left(y^{+}\right)$is always maintained between 30 and 100, as requirement of the logarithmic law of wall for the near-wall zone. Second-order accurate central difference scheme (CDS) coupled with the deferred correction procedure ${ }^{21}$ combining 10 per cent of upwind fluxes and 90 per cent of CDS fluxes was used for the computation. The free-stream turbulence level and the eddy viscosity were assumed to be 1 per cent and 10 times the laminar viscosity respectively.

To validate the present computation results, resistance tests were carried out for the hull model in the high-speed towing tank at Naval Science and Technological Laboratory (NSTL), Visakhapatnam. The tests were conducted at NSTL on $1 / 24^{\text {th }}$ scaled down fibre-reinforced plastic (FRP) models. The models were tested for the bare hull resistance characteristics corresponding to a full-scale draft of $5.05 \mathrm{~m}$. The model is towed using R-47 dynamometer attached to the carriage. The photograph of the hull model with the sonar dome as an integral part is shown in Fig. 7(a).

For code validation, the sonar dome was physically separated from the hull by cutting the model at appropriate location of the dome. The separated dome portion of the hull was connected to the towing carriage through a load cell for the measurement of horizontal forces. The dome was connected to the hull by flexible watertight nylon strips which do not allow any transmission of forces to the remaining part of the hull during the test.

Computed surface pressure contours over the hull surface at a vessel speed of 30 knots are shown in Fig. 7(b). In the zone very close to the bow-end, the flow, in general, bends smoothly along the hull surface after hitting the bow-end edge as a stagnation line. In the stream-wise direction, the flow was observed to remain attached all through the length of the hull considered. The high-pressure region near the stagnation line and the low-pressure zone near the bulge of the sonar dome due to local acceleration were clearly visible in the surface pressure contours. The contours of longitudinal velocity $\left(U / U_{\text {vessel }}\right)$ are shown in Fig. 7(c) at a typical dome cross-section of $X / L=0.0794$ where the bulge due to the dome was maximum. Figure 7(d) compares the computed drag force in Newtons on the sonar dome surface to the corresponding towing tank measurement data ${ }^{26}$ directly, for three different vessel speeds. Reasonable agreement, observed between the computed results and the measurement data, confirms the adequacy of the mathematical model and the numerical accuracy of the present URANS algorithm used for the computation of the three-dimensional turbulent flow field past a ship hull.

\section{CONCLUSIONS}

A fully implicit, second-order accurate pressure-based Navier-Stokes solver in generalised body-fitted non-orthogonal coordinate system, has been developed at the CTFD Division, NAL, Bangalore, for time-accurate calculation of incompressible turbulent flow in or around arbitrary shaped configurations. The algorithm is also parallelised efficiently using the domain decomposition method, coupled to multi-block structured grid for handling complex configuration. The code validation studies have demonstrated the accuracy and adequacy of the spatial and temporal discretisation schemes used, the handling of moving boundaries in an inertial frame of reference, and the proper implementation of parallelisation of the algorithm in a multi-block structured grid environment. For turbulent flows, the URANS approach coupled to variety of linear eddy viscosity-based turbulence models with special near-wall treatments are found to be reasonably accurate for prediction of turbulent boundary layer flows with mild flow separation under moderate adverse pressure gradients. Work is in progress to incorporate nonlinear Eddy viscosity-based models for URANS as well as Large Eddy simulation with dynamic SGS models to achieve better accuracy. Capabilities of the code are being further enhanced to handle multiphysics problems in future incorporating models for multi-phase flows, freesurface flows and reacting flows.

\section{ACKNOWLEDGEMENTS}

The authors wish to thank the Director, Naval Science and Technological Laboratory, Visakhapatnam; Director, ADRDE, Agra and the Hydrodynamics Panel of the Naval Research Board for the financial support provided to this development work through several research projects sponsored to National Aerospace Laboratories (NAL). The authors also wish to thank the Director, NAL, Bangaluru for his kind permission to publish this paper.

\section{REFERENCES}

1. Launder, B.E. \& Spalding, D.B. The numerical computation of turbulent flows. Comp. Meth. Appl. Mech. Engg., 1974, 3, 269-89.

2. Chien, K.Y. Predictions of channel and boundary layer flows with a low Reynolds number turbulence model. AIAA Journal, 1982, 20(1), 33-8.

3. Wilcox, D.C. Turbulence modeling for CFD. DCW Industries.1993.

4. Menter, F.R. Two-equation eddy-viscosity turbulence models for engineering application. AIAA Journal, 1994, 32(8), 1598-605.

5. Spalart, P.R. \& Allmaras, S.R. A one equation turbulence model for aerodynamic flows. AIAA Paper 92-439, 1992.

6. Durbin, P.A. Separated flow computations with the $k-\varepsilon-\overline{v^{2}}$ model. AIAA Journal, 1995, 33(4), 659-64.

7. Majumdar, S.; Rajani, B.N.; Kulkarni, D. \& Mohan, S. RANS computation of low speed turbulent flow in complex configuration. In Proceedings Symposium 
on State-of-the-Art and Future Trends of CFD at NAL, NAL SP 0301, 2003.

8. Majumdar, S. Pressure based Navier Stokes solver for three-dimensional flow in hydrodynamics and low speed aerodynamics applications. In Proceedings of $3^{\text {rd }}$ Asian CFD Conference, Bengaluru, 7-11 December 1998.

9. Majumdar, S.; Rodi, W. \& Zhu, J. Three-dimensional finite volume method for incompressible flows with complex boundaries. J. Fluid Engg. ASME, 1992, 496503.

10. Stone, H.L. Iterative solution of implicit approximations of multidimensional partial differential equations. SIAM J. Num. Anal., 1968, 5, 530-38.

11. Arakeri, J.H. Closure report of the naval research board project on unsteady flow separation. Department of Mechanical Engineering, Indian Institute of Sciences, Bangaluru, 2002.

12. Majumdar, S.; Rajani, B.N.; Kulkarni, D.S. \& Subrhamanya, M. Unsteady RANS simulation of incompressible flow past a symmetric aerofoil at high angles of attack. In Proceedings of $12^{\text {th }}$ Asian Congress of Fluid Mechanics, Daejeon, Korea, August 2008.

13. Leonard, B.P. A stable and accurate convective modelling procedure based on quadratic interpolation. Comp. Meth. Appl. Mech. Engg., 1979, 19, 59-98.

14. Shur, M.M.; Spalart, P.R.; Squires, K.D.; Strelets, M. \& Travin, A. Three dimensionality in Reynolds-Averaged Navier-Stokes Solutions around two-dimensional geometries. AIAA Journal, 2005, 43(6), 1230-42.

15. Hörner, S. F. Fluid-dynamic drag, Park, NJ, 1958. http:/ $/ \mathrm{members}$.aol.com/hfdy/home/htm.Midland.

16. Rajani, B.N.; Govindaraju, L. \& Majumdar, S. Numerical simulation of incompressible viscous flow past a pitching NACA0012 aerofoil. In Proceedings Seventh Asian CFD Conference, Bangaluru, November 2007.

17. McAlister, K.W. An experimental study of dynamic stall on advanced aerofoil section: Pressure and Force Data. NASA TM-84245, vol.2, Sept. 1982

18. Majumdar, S. \& Rajani, B.N. Numerical computation of flow around aerostats using a pressure-based Navier Stokes solver. J. Aero. Soc. India, 2001, 53(2),pp.

19. Narayan, C.L. \& Srilatha, K.R. Analysis of aerostat configurations by panel method. National Aerospace Laboratory, PD CF0010, August 2000

20. Govindaraju, S.P. Evaluation of Aerodynamic forces and moment characteristics on aerostat model in wind tunne. Department of Aerospace Engineering, Indian Institute of Sciences, Report No. IWTR273, June 1999.

21. Khosla, P.K. \& Rubin, S.G. A diagonally dominant second-order accurate implicit scheme. Computers and Fluids, 1974, 2, 207-09

22. Rajani, B.N. \& Majumdar, S. Numerical simulation of turbulent flow around axisymmetric underwater bodies with appendages using a pressure-based RANS solver. In Proceedings of $3^{\text {rd }}$ Asian CFD Conference, Bangaluru, 7-11 December 1998.
23. Huang, T.; Liu, H.L.; Groves, N.; Forlini, T.; Blanton, J. \& Gowing, S. Measurements of flows over an axisymmetric body with various appendages in a wind tunnel: The DARPA sub-off experimental program. In Proceedings of $19^{\text {th }}$ Symposium on Naval Hydrodynamics, 1994. Seoul, Korea.

24. Fathima, A.; Baldawa, N.S.; Pal, S. \& Majumdar, S. Grid generation for arbitrary 2D configurations using a differential algebraic hybrid method. National Aerospace Laboratory, PD CF9416, 1994.

25. Kulkarni, D.S.; Rajani, B.N. \& Majumdar, S. CFD analysis of turbulent flow around the bow mounted sonar dome of P15A ship hull, NAL PD CF0313, December 2003.

26. Kulkarni, D.S.; Rajani, B.N.; Das, H.N.; Jaykumar, P. \& Majumdar, S. Numerical simulation of turbulent flow past the bow-mounted sonar dome of a ship hull. In Proceedings of $31^{\text {st }}$ National Conference on Fluid Mechanics and Fluid Power, Kolkata, 16-18 December 2004.

\section{Contributor}

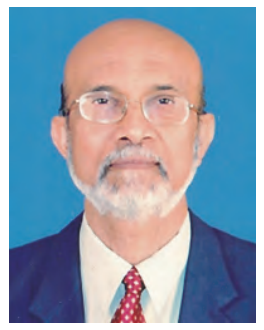

Dr Sekhar Majumdar received his $\mathrm{PhD}$ in Mechanical Engineering in 1981. He was a Senior Scientist at the National Aerospace Laboratories, Bengaluru from 1989 till January 2008 and was the Head of the Computational and Theoretical Fluid Dynamics Division (CTFD) at NAL, Bengaluru. Currently, he is Professor with the Mechanical Engg. Department, NMIT Bengaluru. He worked as a Senior Research Scientist at the Institute for Hydromechanics, University Karlsruhe, Germany and a Senior Visiting Fellow at the Centre for Turbulence Research (2001-2002), Stanford University, California, USA. His research work on CFD for unsteady incompressible flows, turbulence modeling and large eddy simulations. He published about 75 research papers in national/international journals and conference proceedings. He is a Fellow of the Indian National Academy of Engineers and a Fellow of the Aeronautical Society of India. He is a Member, International Committee of Asian Fluid Mechanics Congress, Member Hydrodynamics Panel of NRB and Life Member of the National Society of Fluid Mechanics and Fluid Power, India.

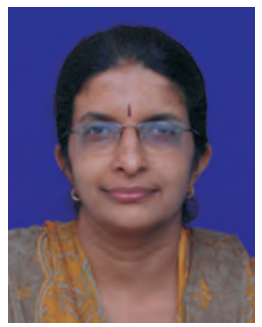

Ms B.N. Rajani obtained her MSc in Mathematics from University of Hyderabad and is currently pursuing her $\mathrm{PhD}$ from NITK, Surathkal (India). She is presently working as a scientist in CTFD Division, NAL, Bengaluru. Her current areas of research include CFD analysis for lowspeed flows, turbulence and transition modelling and large eddy simulation. She has published about 30 research papers in journals and conferences. She has received the NAL Outstanding Performance award in 2002 . 


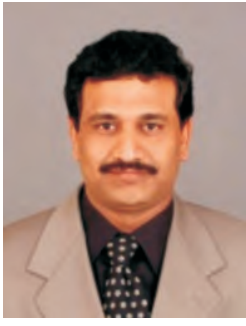

Mr Dattatraya S. Kulkarni obtained his BE (Mechanical Engineering), MTech (Fluid Mechanics and Thermal Sciences) from PDA College of Engineering, Gulbarga and IIT, Kanpur, respectively. Presently, he is working as a scientist in the CTFD division of NAL, Bengaluru. His research areas include CFD analysis of low-speed flows and turbulence and transition modelling. He has more than 17 papers to his credit. He received IEI Young Engineer Award (2008) in Aerospace Engineering.

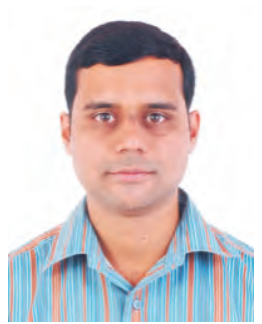

Mr M.B. Subrahmanya obtained his BE (Automobile Engineering) and MTech (Thermal Engineering) from Malnad College of Engineering and NITK, Surathkal, respectively. Presently he is working as a scientist in the CTFD Division at NAL, Bengaluru. His research areas include CFD analysis of low-speed flows and turbulence modelling. He has more than 8 papers to his credit. 